\title{
Briófitas do Município de Poconé, Pantanal de Mato Grosso, MT, Brasil
}

\author{
Paulo Eduardo Aguiar Saraiva Câmara ${ }^{1}$ e Daniel Moreira Vital ${ }^{2}$
}

Recebido em 05/09/2003. Aceito em 11/05/2004

\begin{abstract}
RESUMO - (Briófitas do Município de Poconé, Pantanal de Mato Grosso, MT, Brasil). Sofrendo influências de outros ecossistemas, tais como o Cerrado e a Floresta Amazônica, o Pantanal de Mato Grosso constitui um ecossistema único. Embora sua flora fanerogâmica seja relativamente bem conhecida, a brioflora ainda necessita ser estudada mais profundamente. Fazendo parte da bacia do bio Paraguai e localizado a 94,8km de Cuiabá nas coordenadas 16¹5'24”S e 56³6’24”W, o Município de Poconé possui uma população de cerca de 30.000 habitantes. Foram realizadas coletas briológicas nos anos de 1984, 1999 e 2000, tendo sido encontradas 12 espécies pertencentes a 12 famílias de Bryophyta e 10 espécies distribuídas em duas famílias de Marchantiophyta, sendo Lejeuneaceae a melhor representada, com oito espécies. Foram encontradas cinco novas ocorrências para o região Centro-Oeste: Trichosteleum fluviale (Mitt.) Jaeg., Frullania tetraptera Nees \& Mont., Lejeunea glauscescens Gottsche, Lejeunea calcicola Schuster, Lejeunea caespitosa Lindenb. São ainda citadas nove novas ocorrências para o Estado do Mato Grosso: Hyophila involuta (Hook.) A. Jaeg., Groutiella apiculata (Hook.) Crum \& Steere, Fabronia macroblepharis Schwaegr., Trichosteleum fluviale (Mitt.) Jaeg., Frullania arecae (Spreng.) Gottsche, Frullania tetraptera Nees \& Mont., Lejeunea glauscencens Gottshe, Lejeunea calcicola Schuster e Lejeunea caespitosa Lindenb. Lejeunea calcicola Schuster é citada pela segunda vez para o Brasil.
\end{abstract}

Palavras-chave: Briófitas, Pantanal, Mato Grosso, Poconé

\begin{abstract}
Bryophytes from Pocone county, Pantanal of Mato Grosso, Brazil). Under the influence of outer ecosystems, such as the Cerrado and Amazon rain forest, the Pantanal of Mato Grosso is a unique ecosystem. Otherwise his fanerogamic flora is well known, the bryoflora is yet to be properly discovered. As part of Paraguai river basin and located at $94.8 \mathrm{~km}$ from Cuiabá at $16^{\circ} 15^{\prime} 24^{\prime \prime} \mathrm{S}$ and 56³6'24”W, the county of Poconé has a population around 30,000 hab. Colects were taken in 1984, 1999 and 2000. Among the Division Bryophyte 12 species in 12 familie were founs. In the Division Marchantiophyta 10 species in two families were found. The better represented family was Lejeuneaceae, with eight species. Five new occurrences to the Centro-Oeste region were found: Trichosteleum fluviale (Mitt.) Jaeg., Frullania arecae (Spreng.) Gottsche, Frullania tetraptera Nees \& Mont., Lejeunea glaucescens Gottsche, Lejeunea calcicola Schuster, Lejeunea caespitosa Lindenb. and nine new occurrences to Mato Grosso State were found: Hyophila involuta (Hook.) A. Jaeg., Groutiella apiculata (Hook.) Crum \& Steere, Fabronia macroblepharis Schwaegr., Trichosteleum fluviale (Mitt.) Jaeg., Frullania tetraptera Nees \& Mont., Lejeunea glaucescens Gottshe, Lejeunea calcicola Schuster and Lejeunea caespitosa Lindenb. Lejeunea calcicola Schuster is mentioned for the second time to Brazil.
\end{abstract}

Key words: Bryophytes, Pantanal, Mato Grosso, Poconé

\section{Introdução}

A região Centro-Oeste ainda permanece pouco conhecida em sua brioflora. Embora coletas tenham sido realizadas, poucos trabalhos têm sido publicados. Dentre estes pode-se citar o de Câmara (2002), que fez o primeiro levantamento sistemático de briófitas no Distrito Federal e Câmara (2003). Em especial para a brioflora matogrossense, pode-se mencionar os trabalhos para áreas de cerrado (Guarim Neto \& Yano 1985) e para mata amazônica (Lisboa \& Lisboa 1978), não existindo ainda trabalhos especificos para o pantanal do Mato Grosso.
Sofrendo influências de outros ecossistemas, tais como o Cerrado e a Floresta Amazônica, o Pantanal de Mato Grosso constitui um ecossistema único na região Centro-Oeste. A carência de dados sobre a brioflora do Mato Grosso, em particular do Pantanal, foi o estímulo à realização deste trabalho.

\section{Material e métodos}

Área de Estudo - O Pantanal é uma depressão sazonalmente inundada, integralmente contido na bacia do Alto Paraguai, e abrange uma área de $136.700 \mathrm{~km}^{2}$ nos Estados de Mato Grosso e Mato Grosso do Sul, no

\footnotetext{
1 Missouri Botanical Garden, PoBox 299, Saint Louis, Missouri, 63110. USA. Bolsa CAPES (paulo.camara@mobot.org)/ Universidade de Brasília, Departamento de Botânica

2 Instituto de Botânica, Seção de Briologia e Pteridologia. C. Postal 4005, CEP 01061-970, São Paulo, SP, Brasil
} 
Centro-Oeste brasileiro. A planície do Pantanal apresenta altitude média de $110 \mathrm{~m}$, apesar de se encontrar no centro do continente sul-americano e a mais de $1.500 \mathrm{~km}$ de distância de qualquer linha litorânea. Nessa região tipicamente tropical, a chuva controla o regime dos rios, que, por sua vez, determinam o ciclo das cheias. Entre outubro e março estão os meses chuvosos, responsáveis, em média, por mais de $80 \%$ das precipitações anuais, compreendidas entre 1.000 e $1.200 \mathrm{~mm}$. (Schults \& De-LamonicaFreire 2000). Com exceção dos meses de inverno, junho a agosto, quando a temperatura em certos dias pode ser inferior a $10^{\circ} \mathrm{C}$, as médias de temperatura nas outras épocas do ano são superiores a $25^{\circ} \mathrm{C}$, com máximas ultrapassando os $40^{\circ} \mathrm{C}$. O clima da região é do tipo $\mathrm{Aw}$ segundo a classificação de Köppen. (Schults \& DeLamonica-Freire 2000).

Com 216 anos de existência e localizado a 94,8km de Cuiabá, Mato Grosso, nas coordenadas 16²15’24”S e 56 36'24”'W, o Município de Poconé possui população de cerca de 30.000 habitantes em área de aproximadamente $15.800 \mathrm{~km}^{2}$ (Schults \& DeLamonica-Freire 2000), sendo considerada o “portão" de entrada do Pantanal por ser lá o início da rodovia transpantaneira que liga as cidades de Poconé e Porto Jofre.

Amostragem - Os dados apresentados são resultado de três expedições de coleta ocorridas em 1984, 1999 e 2000. Procurou-se abranger os mais variados substratos e tipos fisionômicos presentes no município e particular no ecossistema pantananeiro. O material foi coletado segundo as orientações de Yano (1984) e encontra-se depositado no herbário da Universidade de Brasília, UB. Quando necessário para identificação foram preparadas lâminas permanentes com solução de Hoyer (Schuster 1969). As identificações foram feitas com o uso de literatura especializada e consulta ao herbário Maria Eneyda P. Kaufmann Fidalgo, do Instituto de Botânica de São Paulo, SP e Universidade de Brasília, UB. As ocorrências novas para o Mato Grosso estão sinalizadas com um asterisco e para o Centro-Oeste com dois.

A distribuição geográfica foi baseada em: Lisboa \& Lisboa (1978), Reese (1979a; 1979b), Griffin III (1979), Yano (1981; 1992; 1994; 1995; 1996), Guarim Neto \& Yano (1985), Yano \& Lisboa (1988), Frahn (1991), Schäfer-Verwimp (1992), Yano \& Mello (1992), Bastos \& Yano (1993), Buck \& Schäfer-Verwimp (1993), Lisboa (1993), Vital \& Visnadi (1994), Yano \& Carvalho (1994), Costa \& Yano (1995), Lisboa \& IlkiuBorges (1995), Visnadi \& Vital (1995; 1997), Schäfer-
Verwimp (1996), Visnadi (2004), Porto et al. (1999), Bastos e Vilas-Bôas-Bastos (2000), Oliveira-e-Silva \& Yano (2000a; 2000b), Ilkiu-Borges (2002a; 2002b; 2002c) e Yano \& Costa (2000), Castro et al. (2002) e Câmara (2003). O sistema de classificação usado para Bryophyta é o de Buck \& Goffinet (2000) e para Marchantiophyta, o de Crandall-Stotler \& Stotler (2000).

\section{Resultados e discussão}

No total foram encontradas 23 espécies de Bryophyta e Marchantiophyta. Dentre as Bryophyta, ocorreram 12 espécies distribuídas entre 12 famílias. Em Marchantiophyta, ocorreram 11 espécies pertencentes a duas famílias, sendo a mais bem representada Lejeuneaceae (oito espécies).

São citadas quatro novas ocorrências para a região Centro-Oeste: Frullania tetraptera Nees \& Mont., Lejeunea glauscescens Gottsche, L. calcicola Schuster e L. caespitosa Lindenb; e oito para o Estado do Mato Grosso: Hyophila involuta (Hook.) A. Jaeg., Groutiella apiculata (Hook.) Crum \& Steere, Fabronia macroblepharis Schwaegr., Frullania arecae (Spreng.) Gottsche, F. tetraptera Nees \& Mont., Lejeunea glauscencens Gottshe, L. calcicola Schuster e Lejeunea caespitosa Lindenb. Lejeunea calcicola Schuster é citada pela segunda vez para o Brasil.

\section{Bryophyta}

Bryaceae

Bryum capillare Hedw., Spec. Musc. 182. 1801.

Descrição e Ilustração: Lisboa (1993).

Material analisado: BRASIL. Mato Grosso: Poconé, 27/V/1984, no solo com cinzas de tronco queimado, ca. $5 \mathrm{~km}$ da Fazenda Boqueirão, Saddi \& Vital 6105-B (UB).

Distribuição geográfica: DF, ES, MT e RO.

Calymperaceae

Syrrhopodon incompletus Scwaegr. Spec. Musc. Frond. Supl. Sec. 1(2): 119. 1824.

Descrição e ilustração: Lisboa (1993).

Material analisado: BRASIL. Mato Grosso: Poconé, 27/V/1984 sobre tronco de palmeira, Acuri, $22 \mathrm{~km}$ ao sul de Poconé, Saddi \& Vital 6117-B, 6118-B (UB); ibidem, sobre Acuri, Fazenda Ypiranga, 20/VII/ 2000, Câmara 706, 712, 713, 716, 719, 720, 721, 
722, 723, 725, 726, 727, 728, 729, 730, 731, 732, 733, 734, 735 (UB).

Distribuição geográfica: AC, AM, AP, BA, GO, MG, MT, PA, PE, PR, RO, RJ, RR, SC e SP.

Erpodiaceae

Erpodium coronatum (Hook. f. Wilson) Mitt. Journ. Linn. Soc. Bot. 12: 403. 1869.

Basiônimo: Anictangium coronatum Hook. F. Wilson. London Journ. Bot. 3: 150. 1844.

Descrição e ilustração: Vital (1980).

Material analisado: BRASIL. Mato Grosso: Poconé, 27/V/1984. Sobre galhos de árvore morta no chão da mata, próximo à fazenda Boqueirão, Saddi \& Vital 6100-B (UB).

Distribuição geográfica: BA, CE, DF, GO, MA, MG, MS, MT, PB, PE, PI, RJ e SP

Fabroniaceae

*Fabronia macroblepharis Schaegr., Sp. Musc. Frond. Suppl. 3(1): 247a. 1828.

Descrição e ilustração: Buck (1998).

Material analisado: BRASIL. Mato Grosso: Poconé, 20/VII/2000. Fazenda Ypiranga, km 10 da rodovia transpantaneira, sobre palmeira Acuri, Câmara 724 (UB).

Distribuição geográfica: GO, MG e SC.

Funariaceae

Jonesiobryum cerradense Vital ex B.H. Allen \& Pursell, Bryologist 94: 441. 1991.

Descrição e Ilustração: Vital (1983).

Material analisado: BRASIL. Mato Grosso: Poconé, 27/V/1984 sobre tronco de Bombacaceae em uma área de cerrado a 9km de Poconé, Saddi \& Vital 6093-B (UB).

Distribuição geográfica: BA, GO, MG, MT, MS e SP.

Hypnaceae

Chryso-hypnum diminutivum (Hampe) Buck, Brittonia 36: 182. 1984.

Basiônimo: Hypnum diminutivum Hampe, Linnaea 20: 86. 1847.

Descrição e Ilustração: Buck (1998).

Material analisado: BRASIL. Mato Grosso: Poconé, 27/V/1984 sobre tronco caído na mata, Saddi \& Vital 6099-B(UB); ibidem, 27/V/1984 sobre tronco de árvore viva, Saddi \& Vital 6103-B, 6104-B (UB).

Distribuição geográfica: AC, AM, AP, DF, GO, ES, MG, MT, PA, PR, RJ, RS, SC e SP.

Leucobryaceae

Octoblepharum albidum Hedw., Spec. Musc. 50. 1801.

Descrição e ilustração: Sharp et al. (1994).

Material analisado: BRASIL. Mato Grosso: Poconé, 20/VII/2000. Fazenda Ypiranga, km 10 da Transpantaneira, sobre tronco de Acuris, Câmara 725, 732, 731, 730, 729, 714 (UB).

Distribuição geográfica: AC, AM, AP, BA, DF, CE, ES, FN, GO, MA, MG, MT, PA, PB, PE, PI, PR, RJ,RO, RR, SE, SC e SP.

Orthotrichaceae

*Groutiella apiculata (Hook.) Crum \& Steere, Bryologist 53: 146. 1950

Basiônimo: Orthotrichum apiculatum Hook., Musci Exot. 1: 45. 1818

Descrição e Ilustração: Sharp et al. (1994).

Material analisado: BRASIL. Mato Grosso: Poconé, 27/V/1984, sobre tronco de árvore viva, Saddi \& Vital 6106-B, 6107-B (UB).

Distribuição geográfica: ES, MS, PA, PE, PR, RJ e SP.

Pottiaceae

*Hyophila involuta (Hook.) A. Jaeg., Ber. Thätigk. St. Gallischen. Naturwiss. Gesel. 1871-1872: 3541873. Basiônimo: Gymnostomum involutum Hook., Musci Exot., 2: 154. 1891.

Descrição e Ilustração: Sharp et al. (1994).

Material analisado: BRASIL. Mato Grosso: Poconé, 12/X/1999. Fazenda Ypiranga, km 10 da Rodovia Transpantaneira. Sobre tronco de palmeira Acuri, na base, Câmara 442 (UB).

Distribuição geográfica: AM, BA, DF, ES, GO, PA, PB, PI, PR, RJ, RO, RS e SP.

Sematophyllaceae

Trichosteleum fluviale (Mitt.) Jaeg., Bericht. Uber. Thati. Galli. Natur. Gessel. 77: 419. 1876.

Basiônimo: Sematophyllum fluviale Mitt. Journ. Linn. Soc. Bot. 12: 493. 1869.

Descrição e Ilustração: Oliveira-e-Silva \& Yano (2000a). 
Material analisado: BRASIL. Mato Grosso: Poconé, 27/V/1984. Sobre tronco caído na mata seca, cerca de $5 \mathrm{~km}$ da fazenda Boqueirão, Saddi \& Vital 6113-B (UB).

Distribuição geográfica: BA, MG, MT, PA, PI e RJ.

Stereophyllaceae

Entodontopsis leucostega (Brid.) Buck \& Ireland, Nova Hedwigia 41: 103. 1985.

Basiônimo: Leskea leucostega Brid. Bryologia Universa 2: 333. 1827.

Descrição e Ilustração: Buck (1998).

Material analisado: BRASIL. Mato Grosso: Poconé, 27/V/1984 na base de tronco vivo na mata seca, próxima à Fazenda Boqueirão, Saddi \& Vital 6097-B (UB); ibidem, 27/V/1984 sobre galhos de árvore morta no chão da mata, próximo à fazenda Boqueirão, Saddi \& Vital 6100-B (UB); ibidem, 27/V/1984 sobre tronco caído na mata, Saddi \& Vital 6104-B (UB); ibidem, 27/V/1984 sobre tronco caído na mata seca a $5 \mathrm{~km}$ SE da Fazenda Boqueirão, Saddi \& Vital 6108-B(UB); ibidem, 27/V/1984 sobre tronco em decomposição no solo, mata seca, Saddi \& Vital 6109-B, 6099-B, 6112-B (UB); ibidem, 27/V/1984 sobre cipó, rodovia transpantaneira, Saddi \& Vital 6116-B (UB); ibidem, 15/IX/1988 Fazenda santo Antônio, estrada Poconé-Boqueirão, Mata seca sobre Myrtaceae, De-Lamonica-Freire 168 (UB); ibidem, 10/VII/2000 sobre tronco de Acuri, Câmara 715 (UB).

Distribuição geográfica: AC, AM, BA, CE, DF, FN, GO, PA, PE, PI, MA, MG, MT, RJ RO e SP.

Thuidiaceae

Cyrto-hypnum schistocalix (Mull. Hall) Buck \& Crum, Contr. Univ. Michigan Herb. 17: 67. 1990.

Basiônimo: Hypnum schistocalix Müll. Hall., Syn. Musc. Frond. 2: 691. 1851.

Descrição e Ilustração: Buck (1998).

Material analisado: BRASIL. Mato Grosso: Poconé, 27/V/1984 sobre tronco em decomposição no solo, mata seca Saddi \& Vital 6112-B (UB).

Distribuição geográfica: AM, PA e MT.

Marchantiophyta

Jubulaceae

*Frullania arecae (Spreng.) Gottsche, Mexik. Leverm. 236. 1863.

Basiônimo: Jungermannia arecae Spreng., Neue
Entdeck. Pflanzenk. 2: 99. 1821.

Descrição e Ilustração: Michel (2001).

Material analisado: BRASIL. Mato Grosso: Poconé, 12/X/1999 Fazenda Ypiranga, km 10 da Rodovia Transpantaneira, sobre tronco de palmeira Acuri, Câmara 442 (UB).

Distribuição geográfica: DF, ES, MG, PR ,RJ, RS e SP.

Frullania ericoides (Nees ex Mart.) Mont., Syn. Hepat. 417. 1845

Basiônimo: Jungermannia ericoides Nees ex Mart., Fl. Brasil. Enum Plant. 1(1): 346. 1833.

Descrição e Ilustração: Michel (2000).

Material analisado: BRASIL. Mato Grosso: Poconé, 27/V/1984 rodovia transpantaneira a cerca de $22 \mathrm{~km}$ ao sul de Poconé, sobre Cipó, Saddi \& Vital 6115-B (UB); ibidem, 20/VII/2000 em tronco de Acuri, Câmara 727, 718 (UB).

Distribuição geográfica: BA PA, PB, PE, DF, ES, FN, GO, MG, MT, RJ, RS, SE e SP.

**Frullania tetraptera Nees \& Mont., Ann. Sci. Nat. Bot. Ser. 2, 9: 47. 1838

Material analisado: BRASIL. Mato Grosso: Poconé, 27-V-1984 sobre cipó na mata secundária, próximo fazenda Boqueirão, Saddi \& Vital 6102-B, 6103-B (UB).

Distribuição geográfica: RJ e SP. Segundo Schiffner \& Arnell (1964), esta espécie seria encontrada nos Estados de RJ e SP. Gradstein \& Costa (2003) sugeriram que o taxa não ocorre no Brasil, sugerindo que o material citado corresponda a F. cuencensis Taylor (Yuzawa 1991). Porém o material pantaneiro não confere. Desta forma, decidiu-se manter o nome $F$. tetraptera

Lejeuneaceae

**Lejeunea caespitosa Lindenb., Syn. Hepat. 382. 1845.

Descrição e Ilustração: Ilkiu-Borges (2002c).

Material analisado: BRASIL. Mato Grosso: Poconé, em tronco de árvore viva, Saddi \& Vital 6098-B, 27-V-1984 (UB); ibidem, 20-VII-2000 sobre tronco de Acuris, Câmara 722, 713, 720, 719, 714, 717, 716 (UB).

Distribuição geográfica: AC, ES, PA, RJ e SP.

**Lejeunea calcicola Schuster, Journ. Elisha Mitchell Sci. Soc. 73: 44. 1957. 
Descrição e Ilustração: Schuster (1980).

Material analisado: BRASIL. Mato Grosso: Poconé, 27/V/1984 sobre tronco caído na mata seca, Saddi \& Vital 6109-B (UB).

Distribuição geográfica: SP. Segunda citação para o Brasil.

Lejeunea trinitensis Lindenb., Syn. Hepat. 381. 1845

Descrição e Ilustração: Schuster (1980).

Material analisado: BRASIL. Mato Grosso: Poconé, 27/V/1984 sobre tronco de árvore viva, Saddi \& Vital 6103-B, 6104-B (UB).

Distribuição geográfica: AC, AM, BAES, GO, MG, MS, MT, PR, RJ e SP.

**Lejeunea glaucescens Gottsche., Syn. Hepat. 378. 1845.

Descrição e Ilustração: Schuster (1980).

Material analisado: BRASIL. Mato Grosso: Poconé, 27/V/1984 sobre tronco de palmeira Acuri, cerca de 22km S de Poconé, Saddi \& Vital 6119-B (UB).

Distribuição geográfica: AC, BA, ES, PA, PE, RJ, RR e SP.

Mastigolejeunea auriculata (Wilson \& Hook.) Schiffner, Hepat. Aust. 129. 1893.

Basiônimo: Jungermannia auriculata Wilson \& Hook., Musci Amer. South Stat. North Am. 170. 1841.

Descrição e Ilustração: Gradstein (1994).

Material analisado: BRASIL. Mato Grosso: Poconé, 27/V/1984 sobre tronco de árvore viva, cerca de $5 \mathrm{~km}$ da fazenda Boqueirão, Saddi \& Vital 6107-B (UB); ibidem, 27/V/1984 sobre cipó em ilha de mata em pleno pantanal cerca de 22km Sul de Poconé, Saddi \& Vital 6114-B (UB).

Distribuição geográfica: AC, AP, BA, ES, GO, MT, PA, PR e SP.

\section{Agradecimentos}

Os autores agradecem ao Dr. Nagib Saddi, in memoriam, que possibilitou as primeiras coletas no Municipio de Poconé; à Faculdade da Terra de Brasília, FTB, que possibilitou as demais coletas; à Bióloga Patrícia Baião Câmara, pelo auxílio nos trabalhos de campo e revisão do texto; ao Dr. Fabian Borghetti, da Universidade de Brasília, pelo apoio logístico. Externam ainda agradecimentos aos proprietários da Fazenda Ypiranga, em Poconé, Dr. João Louzano, Dr. João
Louzano Júnior e Sra. Diorípes Eubanks, pela hospitalidade e infraestrutura.

\section{Referencias bibliográficas}

Bastos, C.J.P. \& Yano, O. 1993. Musgos da zona urbana de Salvador, Bahia, Brasil. Hoehnea 20(1): 23-33.

Bastos, C.J.P. \& Vilas Bôas-Bastos, S.B. 2000. Some new additions to the hepatic flora (Jungermanniophyta) for the state of Bahia, Brazil. Tropical Bryology 18: 1-11.

Buck, W.R. 1998. Pleurocarpous Mosses of West Indies. Memoirs of New York Botanical Garden v.82.

Buck, W.R. 2000. Morphology and classification of mosses. In Shaw \& Goffinet, Bryophyte Biology. Cambridge University Press.

Buck, W.R. \& Schäfer-Verwimp, A. 1993. A reassetment of Schraderobryum (Sematophyllaceae). Boletim do Museu Paraense Emilio Goeldi. Série Botânica 7(2): 645-654.

Câmara, P.S.; Teixeira, R.; Lima, J. \& Lima, J. 2003. Musgos urbanos do recanto das Emas, Distrito Federal, Brasil. Acta Botanica Brasilica 17(4): 507-513.

Castro, N.M.C.F.; Porto, K.C., Yano, O. \& Castro, A.A.J.F. 2002. Levantamento florístico de Bryopsida de cerrado e mata ripícola do Parque Nacional de Sete Cidades, Piauí, Brasil. Acta Botanica Brasilica 16(1): 61-76.

Costa, D.P. \& Yano, O. 1995. Musgos do Município de Nova Friburgo, Estado do Rio de Janeiro, Brasil. Arquivos do Jardim Botânico do Rio Janeiro 33(1): 99-118.

Crandall-Stotler, B. \& Stotler R. 2000. Morphology and classification of the Marchantiophyta. In Shaw, J. \& Goffinet, B, Bryophyte Biology, Cambridge University Press.

Frahm, J.P. 1991. Flora Neotropica. Monograph 54. Dicranaceae: Campylopodioideae, Paraleucobryoideae. Organization for Flora Neotropica, New York, New York Botanical Garden, p.1-238.

Guarim Neto, G. \& Yano, O. 1985. Brioflora da Serra de São Vicente, Mato Grosso. Revista Brasileira de Botanica 8: 199-202

Gradstein S.R. 1994. Flora Neotropica. Monograph. 62. Lejeuneaceae: Ptychanteae Brachiolejeuneae. Organization for Flora Neotropica. New York Botanical Garden. p.1-238.

Griffin III, D. 1979. Guia preliminar para as briófitas freqüentes em Manaus e adjacências. Acta Amazonica 9(3):1-67. Suplemento.

Ilkiu-Borges, A.L. \& Lisboa R.C.L. 2002a. Os gêneros Cyrtolejeunea Evans e Drepanolejeunea Steph. (Lejeuneaceae) na Estação Científica Ferreira Penna (PA) e novas ocorrências. Boletim do Museu Paraense Emilio Goeldi. Série Botânica 18(2): 231-245.

Ilkiu-Borges, A.L. \& Lisboa, R.C.L. 2002b. Leptolejeunea e Rhaphidiolejeunea (Lejeuneaceae) na Estação Científica Ferreira Penna, Pará, Brasil. Acta Amazonica 32(2): 205-215. 
Ilkiu-Borges, A.L. \& Lisboa, R.C.L. 2002c. Os gêneros Lejeunea e Microlejeunea (Lejeuneaceae) na Estação Científica Ferreira Penna, Pará, Brasil. Acta Amazonica 32(2): 541-553.

Lisboa R.C.L. \& Lisboa P.L.B. 1978. Contribuição ao conhecimento da flora do Aripuanã (Mato Grosso) II Musci. Acta Amazonica 8(2): 143-148

Lisboa, R.C.L. 1993. Musgos Acrocárpicos do estado de Rondônia. Boletim do Museu Parense Emilio Goeldi, Coleção Adolpho Ducke.

Lisboa, R. \& Ilkiu-Borges, A. 1995. Diversidade das briófitas de Belém (PA) e seu potencial como indicadoras de poluição urbana. Boletim do Museu Paraense Emílio Goeldi, serie Botânica 11(2): 199-225.

Michel, E.L. 2001. Hepáticas epífitas sobre o pinheiro brasileiro no Rio Grande do Sul. Porto Alegre, Ed. da Universidade, UFRGS.

Oliveira-e-Silva, M.I. \& Yano, O. 2000a. Musgos de Mangaratiba e Angra dos Reis. Rio de Janeiro, Brasil. Boletim do Instituto de Botânica 14: 1-137.

Oliveira-e-Silva M.I. \& Yano O. 2000b. Anthocertotophyta e Hepatophyta de Mangaratiba e Angra dos Reis. Rio de Janeiro, Brasil. Boletim do Instituto de Botânica 13: 1-102.

Porto, K.C.; Gradstein, S.R.; Yano, O.; Germano, S.R. \& Costa, D.P. 1999. New or interesting records of Brazilian bryophytes. Tropical Bryology 17: 39-45.

Reese, W.D. 1979a. New records of Calymperaceae in the Americas. Lindergia 5: 96-98.

Reese, W.D. 1979b. Calymperaceae (Musci) from western Amazonia: Brazil and Bolivia. Bryologist 82(4): 559-563.

Schäfer-Verwimp, A. 1992. New or interesting records of Brazilian Bryophytes, III. Journal of Hattori Botanical Laboratory 71: 55-68.

Schäfer-Verwimp, A. 1996. New or interesting records of Brazilian Bryophytes, V. Candollea 51: 283-301.

Schults, F.P. \& De-Lamonica Freire, E.M. 2000. Desmídias (Chlorophyta, Zygnemaphyceae) do Pantanal de Poconé, Mato-Grosso, Brasil. Diversidades 1: 111-123.

Schuster, R.M. 1969. The hepaticae and anthocerotae of North America East of Hundredt meridian. New York, Columbia Press.

Schuster, R.M.1980. The hepaticae and anthocerotae of North America East of Hundredt meridian. New York, Columbia Press.
Sharp, A.J.; Crum, H.A. \& Eckel, P. 1994. The moss flora of Mexico. Memoirs of the New York Botanical Garden v.69. New York, New York Botanical Garden.

Visnadi, S.R. \& Vital, D.M. 1995. Bryophytes from restinga in Setiba State Park, Espirito Santo State, Brazil. Tropical Bryology 10: 69-74.

Visnadi, S.R. \& Vital, D.M. 1997. Bryophytes from greenhouses of the Institute of Botany, São Paulo, Brazil. Lindbergia 22(1): 44-46.

Visnadi, S.R. 2004. Briófitas de praia do Estado de São Paulo, Brasil. Acta Botanica Brasilica 18(1): 91-97.

Vital, D.M. 1980. Erpodiaceae (Musci) do Brasil. Dissertação de Mestrado. Universidade Estadual de Campinas.

Vital, D.M. 1983. Two new species of Jonesiobryum (Musci) from the brazilian cerrado regions. Journal of Bryology 12: 383-391.

Vital, D.M. \& Visnadi, S.R. 1994. Bryophytes of Rio Branco municipality, Acre, Brasil. Tropical Bryology 9: 69-74.

Yano, O. 1981. A Checklist of Brazilian mosses. The Journal of the Hattori Botanical Laboratory 50: 279-456.

Yano, O. 1984. Briófitas. Pp. 27-30. In: O. Fidalgo (coord.). Técnicas de coleta, preservação e herborização de Material botânico. Instituto de Botânica, Série Documentos.

Yano, O. 1992. Novas Localidades de musgos nos estados do Brasil. Acta Amazonica 22(2): 197-218.

Yano, O. 1994. Briófitas da Serra da Itabaiana, Sergipe, Brasil. Acta Botanica Brasilica 8(1): 45-57.

Yano, O. 1995. A new additional annotated checklist of Brazilian bryophytes. The Journal of the Hattori Botanical Laboratory 78: 137-182.

Yano, O. 1996. A Checklist of brasilian mosses. Boletim do Instituto de Botânica 10: 47-232.

Yano, O. \& Carvalho, A.B. 1994. Musgos do Manguesal do Rio Itanhaém, Itanhaém, São Paulo. Pp. 362-366. In: III Simpósio de Ecossistemas da Costa Brasileira, Serra Negra, SP. São Paulo, ACIESP, v.1.

Yano, O. \& Lisboa, R.C.L. 1988. Briófitas do território Federal do Amapá, Brasil. Boletim do Museu Paraense Emílio Goeldi, Serie Botanica 8(1): 55-80.

Yano, O. \& Mello, Z.R. 1992. Briófitas novas para o estado de Roraima, Brasil. Acta Amazonica 22: 23-50.

Yano, O. \& Costa, D.P. 2000. Briófitas dos estados de Goiás e Tocantins, Coleção Rizzo. Criptógamas: Briófitas, v.5. 33p.

Yuzawa, Y. 1991. A monograph of subg. Chonanthelia of genus Frullania (Hepaticae) of the world. Journal of the Hattori Botanical Laboratory 70: 181-291. 